\title{
Sleeping, Smoking, and Kidney Diseases: Evidence From the NHANES 2017-2018
}

\author{
Chia-Chao Wu ${ }^{1,2}$, Han-En Wang ${ }^{1}$, Yi-Chun Liu ${ }^{3}$, Cai-Mei Zheng ${ }^{4,5,6}$, Pauling Chu ${ }^{1}$, \\ Kuo-Cheng Lu ${ }^{7}$, Chi-Ming Chu ${ }^{3,8,9,10,11}$ and Yu-Tien Chang ${ }^{3 *}$
}

'Division of Nephrology, Department of Internal Medicine, National Defense Medical Center, Tri-Service General Hospital, Taipei, Taiwan, ${ }^{2}$ National Defense Medical Center, Department and Graduate Institute of Microbiology and Immunology, Taipei, Taiwan, ${ }^{3}$ School of Public Health, National Defense Medical Center, Taipei City, Taiwan, ${ }^{4}$ Division of Nephrology, Department of Internal Medicine, Shuang Ho Hospital, Taipei Medical University, New Taipei City, Taiwan, ${ }^{5}$ TMU Research Centre of Urology and Kidney, Taipei Medical University, Taipei, Taiwan, ${ }^{6}$ Department of Internal Medicine, School of Medicine, College of Medicine, Taipei Medical University, Taipei, Taiwan, ${ }^{7}$ Division of Nephrology, Department of Medicine, School of Medicine, Fu-Jen Catholic Hospital, Fu-Jen Catholic University, New Taipei City, Taiwan, ${ }^{8}$ Department of Surgery, National Defense Medical Center, Songshan Branch of Tri-Service General Hospital, Taipei City, Taiwan, ${ }^{9}$ Division of Biostatistics and Informatics, Department of Epidemiology, National Defense Medical Center, School of Public Health, Taipei, Taiwan, ${ }^{10}$ Department of Public Health, China Medical University, Taichung City, Taiwan, ${ }^{11}$ Department of Public Health, Kaohsiung Medical University, Kaohsiung City, Taiwan

\section{OPEN ACCESS}

Edited by:

Carmen Tzanno-Martins,

Hospital Alemão Oswaldo Cruz, Brazil

Reviewed by:

Dirceu Silva,

Clinical Hospital of Porto Alegre, Brazil Andrea Abreu,

University of São Paulo, Brazil

*Correspondence:

Yu-Tien Chang

greengarden720925@gmail.com

Specialty section:

This article was submitted to

Nephrology,

a section of the journal

Frontiers in Medicine

Received: 21 July 2021 Accepted: 30 August 2021 Published: 28 September 2021

Citation:

Wu C-C, Wang H-E, Liu Y-C,

Zheng $C-M$, Chu P, Lu K-C, Chu C-M and Chang Y-T (2021) Sleeping, Smoking, and Kidney Diseases:

Evidence From the NHANES

2017-2018. Front. Med. 8:745006.

doi: 10.3389/fmed.2021.745006
Study Objectives: Smoking and sleep are modifiable factors associated with the chronic kidney diseases. However, the interaction of smoking and sleep on the renal function are still unclear. Therefore, we aimed to evaluate the interactive impacts of smoking and sleep on the renal function.

Methods: Data were obtained from the National Health and Nutrition Examination Survey. The study population were categorized into nine subgroups by smoking (smoking every day, sometimes, and non-smokers recently) and sleep duration (short duration $\leq$ $6 \mathrm{~h}$, normal duration $6-9 \mathrm{~h}$, and longer duration $\geq 9 \mathrm{~h}$ on the weekdays).

Results: The study group with a short sleep duration had significantly higher serum cotinine and hydrocotinine levels compared with the other two sleep groups. After adjusting the demographic characteristics (age, race, body mass index, and marital status), sleep quality (snoring or breathing cessation), and comorbidities (diabetes mellitus, hypertension, high cholesterol, anemia, congestive heart failure, coronary heart disease, and stroke), non-smokers with short or long sleep duration had significant lower estimated glomerular filtration rate (eGFR) levels than the study group who smoked every day and slept $\leq 6 \mathrm{~h}$. The effects of sleep duration on eGFR levels varied with smoking status. For the study group smoking every day, eGFR levels increased as sleep duration decreased, whereas for the study group smoking sometimes, eGFR levels increased as sleep duration increased. The U-shaped effects of eGFR levels were observed among non-smokers whose normal sleep duration was associated with better eGFR levels. Normal sleep duration was an important protective factor of the renal function for non-smokers than smokers.

Conclusions: The effects of sleep duration on eGFR levels varied with smoking status. Normal sleep duration was a protective factor and more crucial for non-smokers than for smokers.

Keywords: sleep, smoking, kidney function, NHANES, eGFR 


\section{INTRODUCTION}

Chronic kidney diseases (CKDs) represent a heavy burden on the healthcare system because of the increasing number of patients, high risk of progression to end-stage renal disease, and poor prognosis with respect to morbidity and mortality (1). Sleep and smoking are two main modifiable factors of CKDs (2). Sleep plays an important role in every aspect of physiology. Sleep reduction has become highly prevalent owing to access to artificial indoor lighting, smartphones, and daily living activities. A populationbased study showed that $22.3 \%$ of men and $28.9 \%$ of women aged $\geq 16$ years told their doctors that they had trouble sleeping (3). Short sleep and long sleep duration (4) as well as poor objective sleep quality have been shown to be associated with the lower estimated glomerular filtration rate (eGFR) and CKD development (5-7).

Smoking is a leading cause of preventable deaths worldwide (8), and increases the risk of developing CKDs (9). The association of longer smoking duration with a higher risk of progression of CKDs was evident particularly in patients with eGFR $<45 \mathrm{ml} / \mathrm{min} / 1.73 \mathrm{~m}^{2}$ and proteinuria $\geq 1.0 \mathrm{~g} / \mathrm{g}$. By contrast, the risk of adverse kidney outcomes decreased with longer smoking-free periods among former smokers (10).

Smoking and sleep problems have been demonstrated to have a reciprocal relationship with each other $(10,11)$. A strong relationship between smoking and subsequent sleep problems was observed in adolescents; this relationship was independent of demographics, snoring, or sleep apnea (SA), body mass index (BMI), depressive symptoms, alcohol use, and soda consumption (12). However, to the best of our knowledge, no study has evaluated the interaction effect of smoking and sleep duration on kidney function. Therefore, we aimed to evaluate the interactive impacts of smoking and sleep on renal function using datasets from the National Health and Nutrition Examination Survey (NHANES).

\section{METHODS}

\section{Data Source}

The National Health and Nutrition Examination Survey (13) is a program of studies designed to assess the health and nutritional status of adults and children in the United States. All the participants provided informed written consent for the study, which was approved by the Ethics Review Board of the National Center for Health Statistics. We used the NHANES datasets from 2017 to 2018, including all the cases of demographic variables (DEMO_J), questionnaire data of smoking and cigarette use (SMQ_J), sleep disorders (SLQ_J), laboratory data of albumin and creatinine-urine (ALB_CR_J), cotinine and hydroxycotinine-serum (COT_J), standard biochemistry profile (BIOPRO_J), blood pressure and cholesterol (BPQ_J), diabetes (DIQ_J), and medical conditions (MCQ_J).

\section{Chronic Kidney Disease Epidemiology Collaboration Equations for eGFR}

The R package of "CKDEpi.creat" (14) and parameters of serum creatinine, sex, age, and ethnicity were used to calculate
eGFR using the CKD-EPI equation. The CKD-EPI equation is expressed as a single equation as follows: GFR $=141 \times \mathrm{min}$ $\left(\mathrm{S}_{\mathrm{Cr}} / \kappa, 1\right)^{\alpha} \times \max \left(\mathrm{S}_{\mathrm{Cr}} / \kappa, 1\right)^{-1.209} \times 0.993^{\text {age }} \times 1.018$ [if female] $\times 1.159$ [if black], where $\mathrm{S}_{\mathrm{Cr}}$ is the standardized serum creatinine in $\mathrm{mg} / \mathrm{dl}, \kappa$ is 0.7 for women and 0.9 for men, a is -0.329 for women and -0.411 for men, min indicates the minimum of $S_{C r} / \kappa$ or 1 , and max indicates the maximum of $S_{C r} / \kappa$ or 1 (15). eGFR values are presented in $\mathrm{ml} / \mathrm{min} / 1.73 \mathrm{~m}^{2}$.

\section{Statistical Analysis}

Statistical analysis was performed using $\mathrm{R}$ version 4.0.2 (16). The testing index distribution was skewed; hence, we used the R package "bestNormalize" (17) to normalize the data. The testing index of blood urea nitrogen $(\mathrm{mmol} / \mathrm{l})$ was normalized using the center scale transformation whereas that of uric acid $(\mu \mathrm{mol} / \mathrm{l})$ was normalized using square root transformation. The levels of serum creatinine $(\mu \mathrm{mol} / \mathrm{l})$, urine creatinine $(\mu \mathrm{mol} / \mathrm{l})$, albumincreatinine ratio $(\mathrm{mg} / \mathrm{g})$, urine albumin $(\mu \mathrm{g} / \mathrm{ml})$, serum cotinine $(\mathrm{ng} / \mathrm{ml})$, and serum hydroxycotinine $(\mathrm{ng} / \mathrm{ml})$ were normalized using log transformation. The descriptive statistics of the testing index are presented as non-normalized figures for clinical use. Transformed figures were used in the multivariable linear regression models. We performed univariable and multivariable linear regression analyses to determine whether sleep and smoking are associated with renal function while controlling for demographic characteristics (age, gender, and marriage), body measurement, marital status, sleep quality, and comorbidities. Variables that were significant in the univariable models were included in the multivariable analyses. The study population was divided into nine subgroups based on sleep duration on weekdays ( $\leq 6,6-9$, and $\geq 9 \mathrm{~h}$ ) and smoking (smoking every day, smoking sometimes, and never smoking recently) for sensitivity analysis. Forest plots were used to present the difference in eGFR levels among the nine subgroups.

\section{RESULTS}

\section{Risk Factors of Kidney Diseases}

The significant risk factors of kidney diseases were as follows: (1) demographic characteristics: male, older age, non-Hispanic white race, higher BMI and widowed/divorced or separated status; (2) sleep quality: frequent snoring or breath cession; (3) smoking: higher serum cotinine and hydrocotinine levels, older age at the start of smoking cigarettes regularly, no smoking recently, smoking since waking for 6-30 min, higher number of smoking days, number of cigarettes in the past 30 days; (4) comorbidities: hypertension, high cholesterol levels, diabetes mellitus, failing kidneys, anemia, congestive heart failure, coronary heart disease, stroke, chronic obstructive pulmonary disease, and malignancy. Blood urea nitrogen, serum creatinine, uric acid, and the albumin-creatinine ratio were negatively associated with eGFR levels (Table 1).

\section{Baseline Characteristics and the Impacts of Sleep Duration on the Renal Functions}

The normal sleep duration group (nmSleep) was the youngest (33.2 \pm 24.3 years) and had the lowest BMI on average (25.6 \pm 
TABLE 1 | Univariable linear regression of the renal function.

eGFR (ml/min/1.73 $\left.\mathrm{m}^{2}\right)$

B

p-values

\section{Demographic characteristics}

Female (ref: male)

Age (year)

Race (ref: Mexican American)

Other Hispanic

Non-Hispanic white

Non-Hispanic black

Other Race-including multi-racial

Weight (kg)

Height (cm)

BMI (kg/m²)

Marital status (ref: Married or living with partner)

Never married

Widowed, divorced, or separated

$-4.24$

$-5.43$

$-0.21$

$-0.20$

$-0.64$

Renal function

Blood urea nitrogen ( $\mathrm{mmol} / \mathrm{L})$

Creatinine, refrigerated serum (umol/L)

Uric acid (umol/L)

Albumin creatinine ratio $(\mathrm{mg} / \mathrm{g})$

12.55

$-11.51$

$-7.76$

$-0.40$

$-0.10$

$-0.01$

\section{Metabolites of nicotine}

Serum cotinine $(\mathrm{ng} / \mathrm{mL})$

Serum hydrocotinine ( $\mathrm{ng} / \mathrm{mL}$ )

Sleep quality

Sleep duration on weekdays (h)

Rarely-1-2 nights a week

Occasionally-3-4 nights a week

$$
-8.60
$$

How often do you snort or stop breathing (ref: never)

Rarely-1-2 nights a week

Occasionally-3-4 nights a week

Frequently -5 or more nights a week

\section{Smoking status}

Age started smoking cigarettes regularly

Do you now smoke cigarettes? (ref: Every day)

Some days

Not at all

\# days smoked cigarettes during past 30 days

Average \# cigarettes/day during past 30 days

$-1.81$

$-0.42$

$-0.21$

Tried to quit smoking

Comorbidities

High blood pressure

High cholesterol

$-21.40$

$-17.85$

$\star \star \star$

$\star \star \star *$

$\star \star *$

$\star \star \star *$

Diabetes (ref: Yes)

No

$\star \star \star *$

$\star \star \star$

0.60

*

$\star \star \star$

$\star \star \star$

*

$\star \star \star *$

$\star \star \star *$

**

0.63

$\star \star \star$

0.40

$\star \star \star *$

*

0.60

$\star \star \star$

$\star \star \star$

$\star \star \star$ 


\begin{tabular}{|c|c|c|}
\hline & \multicolumn{2}{|c|}{ eGFR (ml/min/1.73 m²) } \\
\hline & B & $p$-values \\
\hline Borderline & 10.27 & 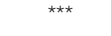 \\
\hline Failing kidneys & -37.57 & $\star \star \star ~$ \\
\hline Anemia & -7.14 & 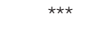 \\
\hline Congestive heart failure & -28.80 & 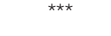 \\
\hline Coronary heart disease & -23.38 & 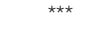 \\
\hline Stroke & -21.78 & 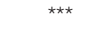 \\
\hline Chronic obstructive pulmonary disease & -16.74 & 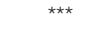 \\
\hline Malignancy & -20.38 & 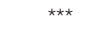 \\
\hline
\end{tabular}

${ }^{\star} p<0.05,{ }^{\star \star} p<0.01,{ }^{\star \star \star} p<0.001$. Ref, reference group.

$8.14 \mathrm{~kg} / \mathrm{m}^{2}$ ). The primary marital status of nmSleep was married or living with partners $(60.5 \%)$ compared with the other two sleep groups with less or more sleep duration (abbreviation: lessSleep and moreSleep). Except for malignancy, nmSleep had a lower prevalence of hypertension, high cholesterol, DM, failing kidneys, anemia, congestive heart failure, coronary heart disease, heart attack, stroke, and COPD than the other two sleep groups (Table 2).

Renal function was associated with sleep duration. nmSleep and moreSleep had higher eGFR levels. nmSleep had the lowest blood urea nitrogen, the lowest serum creatinine, and a lower uric acid among the three sleep groups. No difference was found in the albumin-creatinine ratio. Taken together, nmSleep had the best renal function. The U-shaped effects of the renal function levels were observed among the sleep duration groups (Table 2). Significantly higher cotinine $(75.6 \pm 152 \mathrm{ng} / \mathrm{ml}, p<0.001)$ and hydrocotinine $(30.9 \pm 82 \mathrm{ng} / \mathrm{ml}, p<0.001)$ levels were observed in lessSleep compared with the other two sleep groups (Table 2; Figure 1A).

\section{Baseline Characteristics and the Impacts of Smoking on the Renal Functions}

The non-smoker group (noSmoking) was predominantly male (63.5\%), non-Hispanic White (45.7\%), married or living with partners, and older (58.8 \pm 17 years) and had higher BMI (30.8 $\pm 7.39 \mathrm{~kg} / \mathrm{m}^{2}$ ). The prevalence of hypertension, high cholesterol, DM, failing kidneys, anemia, congestive heart failure, coronary heart disease, and malignancy was higher in noSmoking than other smoking groups of smoking every day (edSmoking) or smoking sometimes (stSmoking). The COPD prevalence was the highest in edSmoking (Table 3).

The non-smoker group had worse indices of the renal function than the other two smoking groups, but no difference was found in albumin-creatinine ratio levels (Table 3). The cotinine and hydrocotinine levels were positively associated with smoking frequency (Table 3; Figure 1B). They were highly correlated with each other as well with the Pearson correlation r $0.85(p<0.001)$ (Figure 1C).

\section{Sleep Quality and Smoking Characteristics of the Study Population}

Study population with sleep duration $<6 \mathrm{~h}$ tended to had worse sleep qualities of snoring, breath cessation, and having trouble in sleeping. This sleep subgroup was more likely to smoke every day, smoke at least 100 cigarettes in life, and smoke within $30 \mathrm{~min}$ after waking up (Table 4). Over half percent of nmSleep and moreSleep did not smoke recently. The average sleep duration of edSmoking, stSmoking, and noSmoking were $7.42 \pm 1.91$, $7.50 \pm 1.77$, and $7.66 \pm 1.66 \mathrm{~h}$, respectively. There was no significant difference in the sleep qualities among the three smoking subgroups (Table 5). The U-shaped effects of cotinine levels among the three sleep subgroups were only observed in the study population smoked sometimes that normal sleep duration group had lower cotinine levels (Figure 1D).

\section{The Interactive Impacts of Sleep Duration and Smoking on the Renal Function}

The baseline eGFR levels of the study population who smoked every day or were sometimes similar and higher than the nonsmokers (Figure 2A). Although the interaction term of smoking and sleep duration was not significant in the multivariable linear regression analysis (data not shown), the patterns of eGFR levels in the nine subgroups varied. For the study population who smoked every day, the longer the sleep time, the lower the eGFR levels. For non-smokers, the reverse U-shaped effects of eGFR levels were observed. Non-smokers with normal sleep duration had higher eGFR levels as compared with the other two sleep groups. For the study population who smoked sometimes, the longer sleep duration, the higher were the eGFR levels (Figure 2B).

After the adjustment of the demographic characteristics (age, race, BMI, and marital status), sleep quality (snoring or breathing cessation), and comorbidities (diabetes, high blood pressure, high cholesterol, anemia, congestive heart failure, coronary heart disease, and stroke), only noSmokinglessSleep and noSmoking-moreSleep had significantly lowered eGFR levels compared with edSmoking-lessSleep. The Ushaped effect of sleep duration on the renal function was 
TABLE 2 | Baseline characteristics of study population grouped by sleep duration.

\begin{tabular}{|c|c|c|c|c|}
\hline & \multicolumn{4}{|c|}{ Sleep duration on weekdays } \\
\hline & $\begin{array}{c}\leq 6 h \\
(n=1,118)\end{array}$ & $\begin{array}{c}6-9 \mathrm{~h} \\
(n=5,652)\end{array}$ & $\begin{array}{c}\geq 9 \mathrm{~h} \\
(n=1,499)\end{array}$ & $p$-values \\
\hline \multicolumn{5}{|l|}{ Demographic characteristics } \\
\hline Female & $512(45.8 \%)$ & $2,874(50.8 \%)$ & $841(56.1 \%)$ & 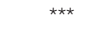 \\
\hline Age (year) & $48.5(17.8)$ & $33.3(24.3)$ & $49.1(22.0)$ & 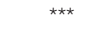 \\
\hline Race & & & & 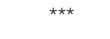 \\
\hline Mexican American & $136(12.2 \%)$ & $848(15.0 \%)$ & $223(14.9 \%)$ & \\
\hline Other Hispanic & $105(9.39 \%)$ & $489(8.65 \%)$ & $140(9.34 \%)$ & \\
\hline Non-Hispanic white & $314(28.1 \%)$ & $1,907(33.7 \%)$ & $538(35.9 \%)$ & \\
\hline Non-Hispanic black & $367(32.8 \%)$ & $1,211(21.4 \%)$ & $335(22.3 \%)$ & \\
\hline Other Race-including multi-racial & $196(17.5 \%)$ & $1,197(21.2 \%)$ & $263(17.5 \%)$ & \\
\hline Weight (kg) & $85.2(24.9)$ & $65.3(30.4)$ & $79.3(22.2)$ & 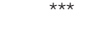 \\
\hline Height (cm) & $167(10.3)$ & $155(22.0)$ & $165(9.90)$ & $* \star \star$ \\
\hline BMI $\left(\mathrm{kg} / \mathrm{m}^{2}\right)$ & $30.3(8.10)$ & $25.6(8.14)$ & $28.9(7.29)$ & 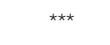 \\
\hline Marital status & & & & ** \\
\hline Married or living with partner & $602(57.6 \%)$ & $1,949(60.5 \%)$ & $701(54.1 \%)$ & \\
\hline Never married & $199(19.0 \%)$ & $569(17.7 \%)$ & $238(18.4 \%)$ & \\
\hline Widowed, divorced, or separated & $245(23.4 \%)$ & $704(21.8 \%)$ & $356(27.5 \%)$ & \\
\hline \multicolumn{5}{|l|}{ Comorbidities } \\
\hline High blood pressure & $424(37.9 \%)$ & $1,181(33.4 \%)$ & $532(35.6 \%)$ & * \\
\hline High cholesterol & $356(32.2 \%)$ & $1,117(31.7 \%)$ & $495(33.2 \%)$ & 0.60 \\
\hline Diabetes & & & & 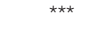 \\
\hline Yes & 154 (13.8\%) & 487 (8.62\%) & $252(16.8 \%)$ & \\
\hline No & $926(82.9 \%)$ & $5,050(89.4 \%)$ & $1,212(80.9 \%)$ & \\
\hline Borderline & $37(3.31 \%)$ & $113(2.00 \%)$ & 34 (2.27\%) & \\
\hline Failing kidneys & $50(4.79 \%)$ & $100(3.10 \%)$ & $73(5.64 \%)$ & *** \\
\hline Anemia & $54(4.84 \%)$ & $177(3.13 \%)$ & $89(5.97 \%)$ & 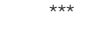 \\
\hline Congestive heart failure & 55 (5.27\%) & $85(2.64 \%)$ & $61(4.72 \%)$ & 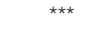 \\
\hline Coronary heart disease & $60(5.74 \%)$ & $121(3.76 \%)$ & $84(6.49 \%)$ & 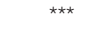 \\
\hline Stroke & 57 (5.47\%) & $118(3.67 \%)$ & $98(7.56 \%)$ & 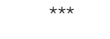 \\
\hline Chronic obstructive pulmonary disease & $71(6.81 \%)$ & $135(4.19 \%)$ & $87(6.72 \%)$ & 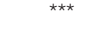 \\
\hline Malignancy & $91(8.71 \%)$ & $334(10.4 \%)$ & $163(12.6 \%)$ & $* \star \star$ \\
\hline \multicolumn{5}{|l|}{ Renal function } \\
\hline Blood Urea Nitrogen (mmol/L) & $5.36(2.28)$ & $5.14(2.04)$ & $5.33(2.26)$ & ** \\
\hline Creatinine, refrigerated serum (umol/L) & $82.6(53.5)$ & $75.1(33.3)$ & $79.6(42.1)$ & 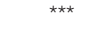 \\
\hline $\mathrm{eGFR}\left(\mathrm{ml} / \mathrm{min} / 1.73 \mathrm{~m}^{2}\right)$ & $95.8(25.5)$ & $103(28.8)$ & $95.0(28.6)$ & 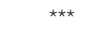 \\
\hline Uric acid (umol/L) & $330(87.8)$ & $320(87.9)$ & $319(88.4)$ & ** \\
\hline Albumin creatinine ratio (mg/g) & $44.0(223)$ & 38.1 (294) & $59.9(365)$ & 0.06 \\
\hline \multicolumn{5}{|l|}{ Metabolites of nicotine } \\
\hline Serum cotinine (ng/mL) & $75.6(152)$ & $30.4(95.9)$ & 49.7 (122) & 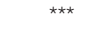 \\
\hline Seum hydrocotinine (ng/mL) & 30.9 (82.0) & $11.7(41.6)$ & $21.4(58.8)$ & 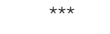 \\
\hline
\end{tabular}

${ }^{*} p<0.05,{ }^{* *} p<0.01,{ }^{* \star *} p<0.001$ using statistical methods of ANOVA and Chi-squared test.

significantly observed in noSmoking that nmSleep had better eGFR than the other two sleep groups (Figure 3). Normal sleep duration was a predominant profactor of the renal function in noSmoking. For the smokers, sleep duration had no significant effect on eGFR. A controversial finding is that noSmokingmoreSleep or noSmoking-lessSleep had lower eGFR than edSmoking-lessSleep in both the univariable and multivariable models (Figure 3).

\section{DISCUSSION}

Accumulating the clinical evidence suggests that cigarette smoking has a negative effect on the renal function, kidney dimensions (18), and CKD development of different etiologies, including DM, and hypertension $(19,20)$. Cigarette smoking is one of the most important modifiable renal risk factors (21). Nicotine, a major tobacco alkaloid, associates smoking with renal 


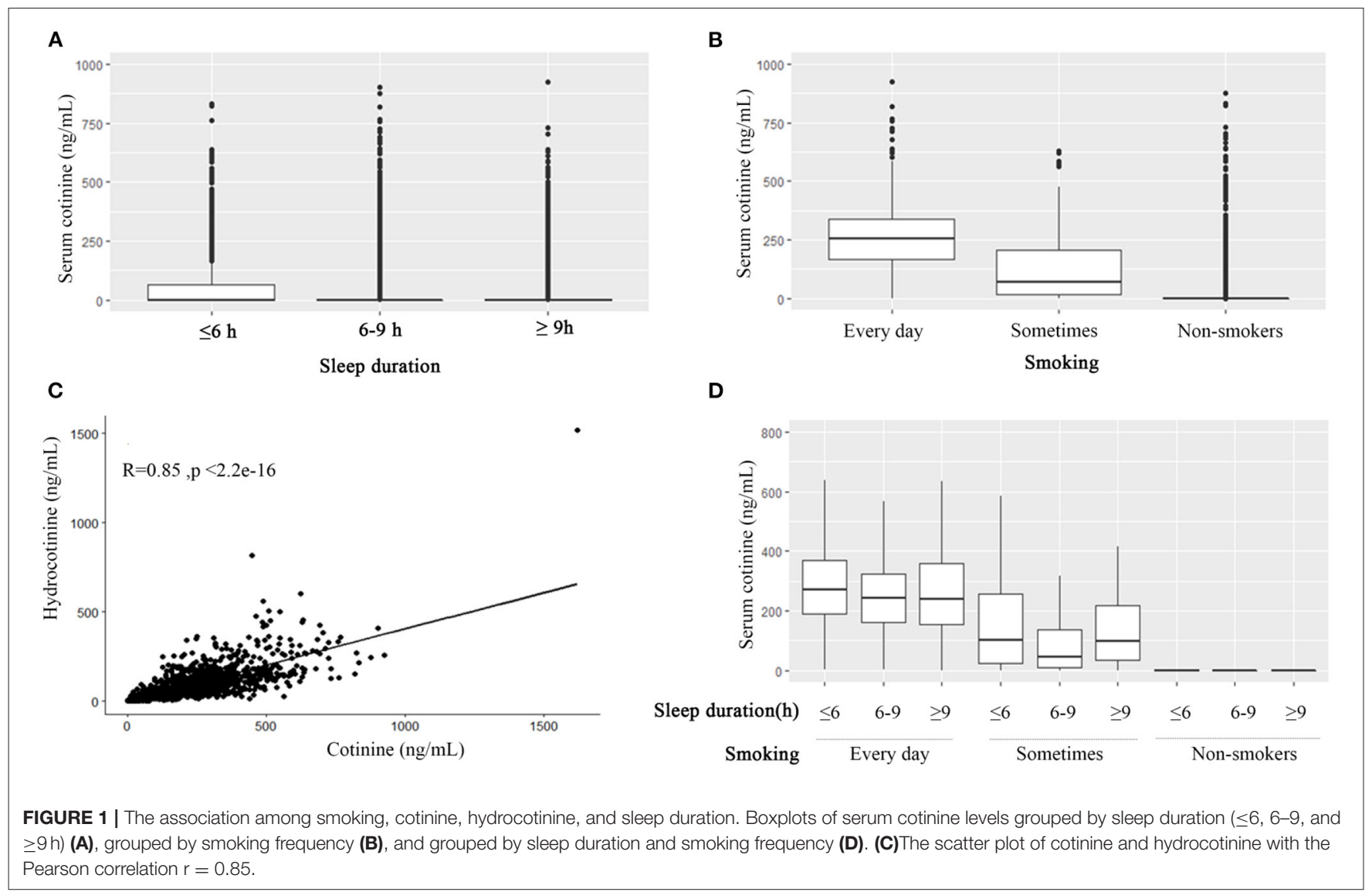

dysfunction $(22,23)$. The risk of adverse kidney outcomes was incrementally higher as a smoking pack-years increased (24). Exposure to nicotine has been strongly shown to enhance renal oxidative stress (22) and kidney failure (25). Chronic exposure to nicotine accelerates the onset and progression of renal diseases in habitual cigarette smokers.

A major pathway of nicotine metabolism is C-oxidation, followed by cotinine; and the subsequent hydroxylation to trans- $3^{\prime}$-hydroxycotinine. Moreover, $85-95 \%$ of the total nicotine uptake is eliminated as cotinine, hydroxycotinine, and glucuronides in the urine $(26,27)$. Cotinine has a longer plasma half-life than nicotine and showed a dose-dependent effect of smoking exposure $(26,28-30)$. This is in line with our finding; smoking frequency and serum cotinine and hydrocotinine levels were positively associated with each other. Therefore, we divided the smoking groups by the self-report of smoking frequency from the NHANES dataset.

The mechanisms of smoking-related renal damage are poorly understood, but the damage is likely caused by vascular and tubular effects (22). Smoking may sensitize the kidney to ischemic insults and perhaps facilitate the progression of acute kidney injury to chronic kidney injury (22). Nicotine increases the severity of renal injury in animal models leading to acute kidney injury, DM, acute nephritis, and subtotal nephrectomy (19). Nicotine stimulates the proliferation and hypertrophy of mesangial cells. Nicotine administration to sham rats increased total proteinuria but not albuminuria, indicating that nicotine directly affects tubular protein reabsorption (31). In humans, nicotine induces transitory increases in blood pressure accompanied by reductions in eGFR and effective renal plasma flow (19).

We found that the serum blood urea nitrogen and creatinine were higher and that eGFR levels were lower in the group of noSmoking than in the group of edSmoking and stSmoking after adjusting for age, race, BMI, marital status, and comorbidities (DM, hypertension, high cholesterol level, congestive heart failure, coronary heart disease, angina/angina pectoris, heart attack, stroke, emphysema, chronic bronchitis, and anemia). This finding is contradictory to the results of most studies (19-25).

In a study involving 28,409 individuals, smokers exhibited a slightly higher creatinine clearance rate than non-smokers at least in men after adjusting for hypertension (32). Moreover, the administration of nicotine to adolescent mice for 4 weeks incited higher oxidative stress and tubular injury than in adult kidney, but it did not modify creatinine levels (33). Does this higher creatinine clearance in smokers signify a better renal function? This increase may reflect a direct effect of smoking on tubular creatinine secretion or interfere with the estimation methods of creatinine. A 24-h urine collection would considerably eliminate any interference between smoking and estimation of creatinine 
TABLE 3 | Baseline characteristics of study population grouped by smoking.

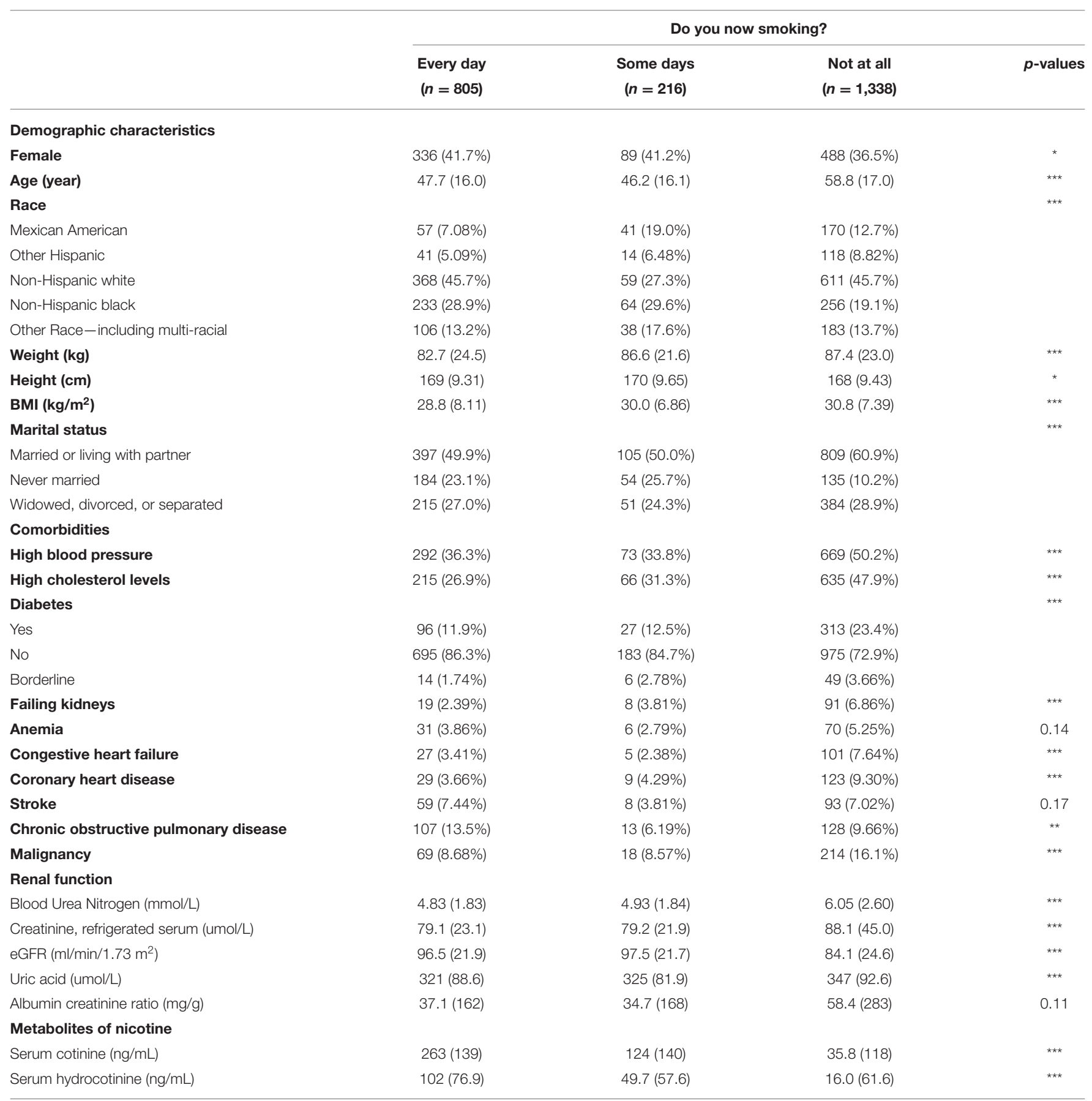

${ }^{*} p<0.05,{ }^{* *} p<0.01,{ }^{* \star *} p<0.001$ using statistical methods of ANOVA and Chi-squared test.

levels (32). The creatinine-based eGFR raises as the smoking amount increases, whereas the cystatin C-based eGFR decreases (34). This finding might indicate that creatinine-based eGFR, which was adopted in this study, may not be an ideal marker to estimate the relationship between smoking and renal function. Current smoking status cannot reflect the history of past exposure to cigarettes, and nicotine tends to be a short-term exposure marker of smoking. This is a limitation of this study. A high proportion of noSmoking individuals may have had a history of long past exposure to smoking cigarettes.

The finding of better renal function in smokers might have some biological plausibility. Lower doses of subacute nicotine administration can enhance renal function (35). Moreover, nicotine has a protective effect against neurotoxic insults and may 
TABLE 4 | Sleep quality and smoking characteristics of study population grouped by sleep duration.

\begin{tabular}{|c|c|c|c|c|}
\hline & \multicolumn{4}{|c|}{ Sleep duration on weekdays } \\
\hline & $\begin{array}{c}\leq 6 h \\
(n=1,118)\end{array}$ & $\begin{array}{c}6-9 \text { h } \\
(n=5,652)\end{array}$ & $\begin{array}{c}\geq 9 h \\
(n=1,499)\end{array}$ & $p$-values \\
\hline \multicolumn{5}{|l|}{ Sleep quality } \\
\hline Sleep duration (h) & $5.23(0.969)$ & $7.54(0.617)$ & $9.74(1.01)$ & $\star \star \star \star ~$ \\
\hline How often do you snore & & & & 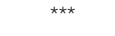 \\
\hline Never & $284(27.9 \%)$ & $925(28.2 \%)$ & $479(34.2 \%)$ & \\
\hline Rarely-1-2 nights a week & $226(22.2 \%)$ & $828(25.2 \%)$ & $316(22.5 \%)$ & \\
\hline Occasionally $-3-4$ nights a week & $185(18.2 \%)$ & $648(19.7 \%)$ & $235(16.8 \%)$ & \\
\hline Frequently -5 or more nights a week & $324(31.8 \%)$ & $881(26.8 \%)$ & $372(26.5 \%)$ & \\
\hline How often do you snore or have breath cessation & & & & 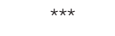 \\
\hline Never & $778(74.0 \%)$ & $2,592(77.6 \%)$ & $1,104(77.7 \%)$ & \\
\hline Rarely $-1-2$ nights a week & $133(12.7 \%)$ & $408(12.2 \%)$ & $158(11.1 \%)$ & \\
\hline Occasionally-3-4 nights a week & $59(5.61 \%)$ & $200(5.98 \%)$ & $102(7.18 \%)$ & \\
\hline Frequently-5 or more nights a week & $81(7.71 \%)$ & $142(4.25 \%)$ & $57(4.01 \%)$ & \\
\hline Having trouble sleeping & $346(30.9 \%)$ & $858(24.2 \%)$ & 417 (27.9\%) & 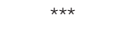 \\
\hline \multicolumn{5}{|l|}{ Smoking status } \\
\hline Smoked at least 100 cigarettes in life (yes/no) & 489 (45.3\%) & $1,293(38.4 \%)$ & 577 (40.8\%) & 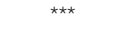 \\
\hline Age started smoking cigarettes regularly (years) & $17.8(6.06)$ & $18.0(6.53)$ & $17.9(6.33)$ & 0.89 \\
\hline Do you now smoke cigarettes & & & & ** \\
\hline Every day & 202 (41.3\%) & 417 (32.3\%) & $186(32.2 \%)$ & \\
\hline Some days & $50(10.2 \%)$ & $120(9.28 \%)$ & $46(7.97 \%)$ & \\
\hline Not at all & 237 (48.5\%) & $756(58.5 \%)$ & 345 (59.8\%) & \\
\hline How soon after waking do you smoke & & & & * \\
\hline$\leq 5 \min$ & $54(27.7 \%)$ & $85(20.3 \%)$ & $56(31.3 \%)$ & \\
\hline $6 \sim 30 \mathrm{~min}$ & $66(33.8 \%)$ & $157(37.5 \%)$ & $48(26.8 \%)$ & \\
\hline$\geq 30 \min$ & $75(38.5 \%)$ & $177(42.2 \%)$ & $75(41.9 \%)$ & \\
\hline \# days smoked cigarettes during past 30 days & $25.6(8.65)$ & $24.2(10.0)$ & $24.8(9.19)$ & 0.16 \\
\hline Average \# cigarettes/day during past 30 days & $11.2(8.06)$ & $11.2(8.77)$ & $10.3(7.53)$ & 0.42 \\
\hline Tried to quit smoking (yes/no) & $126(49.6 \%)$ & $290(53.0 \%)$ & $125(53.4 \%)$ & 0.62 \\
\hline
\end{tabular}

${ }^{\star} p<0.05,{ }^{* \star} p<0.01,{ }^{\star \star \star} p<0.001$ using statistical methods of ANOVA and Chi-squared test.

be of potential therapeutic value in Parkinson's disease (36). In addition, cotinine reduced fear memory and anxiety after fear conditioning and improved working memory in a mouse model of Alzheimer's disease and in a monkey model of schizophrenia (37). Nicotine pretreatment reduced tubular damage (tubular cell apoptosis and proliferative response) due to an innate immune response in animal model experiments (38).

We found that the normal sleep duration of 6-9 h is associated with better eGFR and other renal function indices (blood urea nitrogen, creatinine, and uric acid) compared with the other two sleep duration groups; however, there was no difference in the albumin-creatinine ratio. In agreement with the previous studies, short and long sleep durations have been associated with adverse health outcomes in the general population $(39,40)$ and in patients with CKDs and diabetic kidney disease (DKD) (39, 41, 42). Overall, the cutoffs of normal sleep duration (6-8 or 6-9h) may differ slightly, but the finding remained consistent (42). Physiological evidence indicated that sleep influences kidney function. The genetic risk score for short, but not long, sleep duration was significantly related with a higher risk of CKD stages 3-5 (6).

A poor sleep profile or quality is another important risk factor of increasing CKDs risk (42). SA is a condition that has serious health consequences, has an increased risk of death, and is common in patients with CKDs $(43,44)$ and DKD $(45)$. Obstructive SA-related hypoxia causes several negative systemic effects, including oxidative stress $(46,47)$, inflammation, and sympathetic activation, all of which contribute to the progression of renal disease. In turn, CKD can result in the increased severity of SA by inducing uremic neuropathy and myopathy, altered chemosensitivity, and hypervolemia (43).

Sleeping behaviors and smoking have a reciprocal effect and a moderate correlation with genetics (48). Severe smoking status appears to have a causal effect on the circadian rhythm, and some evidence has shown that insomnia increases smoking heaviness and impedes cessation (48). Indeed, cigarette smoking has been shown to be associated with sleep disturbance via prolonged sleep-onset latency, 
TABLE 5 | Sleep and smoking characteristics of study population grouped by smoking.

\begin{tabular}{|c|c|c|c|c|}
\hline & \multicolumn{4}{|c|}{ Do you now smoking? } \\
\hline & $\begin{array}{l}\text { Every day } \\
(n=805)\end{array}$ & $\begin{array}{l}\text { Some days } \\
\qquad(n=216)\end{array}$ & $\begin{array}{l}\text { Not at all } \\
(n=1,338)\end{array}$ & $p$-values \\
\hline \multicolumn{5}{|l|}{ Sleep quality } \\
\hline Sleep duration (h) & $7.42(1.91)$ & $7.50(1.77)$ & $7.66(1.66)$ & * \\
\hline How often do you snore & & & & 0.14 \\
\hline Never & $203(27.5 \%)$ & $53(27.0 \%)$ & $277(22.8 \%)$ & \\
\hline Rarely-1-2 nights a week & $144(19.5 \%)$ & $49(25.0 \%)$ & $274(22.6 \%)$ & \\
\hline Occasionally-3-4 nights a week & $136(18.4 \%)$ & $31(15.8 \%)$ & 242 (19.9\%) & \\
\hline Frequently -5 or more nights a week & $255(34.6 \%)$ & $63(32.1 \%)$ & $422(34.7 \%)$ & \\
\hline How often do you snort or have breath cessation & & & & 0.59 \\
\hline Never & $516(70.3 \%)$ & $159(77.6 \%)$ & $874(71.0 \%)$ & \\
\hline Rarely $-1-2$ nights a week & $107(14.6 \%)$ & $23(11.2 \%)$ & $174(14.1 \%)$ & \\
\hline Occasionally-3-4 nights a week & $61(8.31 \%)$ & $12(5.85 \%)$ & $95(7.72 \%)$ & \\
\hline Frequently -5 or more nights a week & $50(6.81 \%)$ & $11(5.37 \%)$ & $88(7.15 \%)$ & \\
\hline Having trouble sleeping & $296(36.8 \%)$ & $66(30.6 \%)$ & $464(34.7 \%)$ & 0.21 \\
\hline \multicolumn{5}{|l|}{ Smoking status } \\
\hline Age started smoking cigarettes regularly (years) & $17.9(6.07)$ & $18.9(9.28)$ & $17.8(5.97)$ & 0.07 \\
\hline \multicolumn{5}{|l|}{ How soon after waking do you smoke } \\
\hline$\leq 5 \min$ & 194 (24.9\%) & - & - & \\
\hline $6 \sim 30 \mathrm{~min}$ & $270(34.7 \%)$ & - & - & \\
\hline$\geq 30 \mathrm{~min}$ & $315(40.4 \%)$ & - & - & \\
\hline \# days smoked cigarettes during past 30 days & $29.4(2.99)$ & $11.3(7.15)$ & - & *** \\
\hline Average \# cigarettes/day during past 30 days & $12.8(8.07)$ & $4.13(4.99)$ & - & 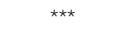 \\
\hline
\end{tabular}

${ }^{\star} p<0.05,{ }^{* \star \star} p<0.001$ using statistical methods of ANOVA and Chi-squared test.

higher dopamine levels, and lower dopamine transporter levels in the cerebrospinal fluid of active smokers (49). The symptoms of cigarette smoking and nicotine dependence were associated with poor sleep quality in young adult smokers (50). Both oral nicotine administration and abstinence led to sleep disturbances in mice (51). In line with these findings, lessSleep individuals were shown to be more likely to smoke and had higher levels of cotinine and hydrocotinine in this study.

Many studies have discussed the individual association of sleep and smoking with the renal function in general, CKDs or DKD population. To the best of our knowledge, this is the first study to discuss the interaction effect of sleep duration and smoking on renal function. We observed that the nonsmokers who had less or more sleep exhibited significantly lower eGFR levels compared with those who smoked every day and slept less. This association was independent of demographic characteristics (age, race, BMI, marital status), sleep quality of snoring or breathing cessation, and comorbidities (high blood pressure, high cholesterol, anemia, congestive heart failure, coronary heart disease, and stroke). This finding contradicts those of many studies that the risk of adverse kidney outcomes was incrementally higher as smoking pack-years increased (18, 19, 24). Even, we evaluated the eGFR of normal or abnormal serum cotinine groups, the average eGFR levels of the abnormal cotinine group were still slightly higher than the normal cotinine group. In noSmoking, the eGFR levels of the study population with normal levels of cotinine or hydrocotinine were lower than or equal to those with abnormal levels of cotinine.

The contradictory findings of smoking and nicotine on adverse kidney outcomes can be attributed to the following factors: (1) information on the nicotine exposure history of dose and length are not available (52), (2) creatinine-based eGFR may not be an ideal marker to estimate the relationship between smoking and the renal function (34), (3) other harmful ingredients from cigarettes have a greater effect on the renal function than nicotine (53), and (4) the non-smoking group possessed more risk factors (older age, male predominance, BMI, higher proportion of non-Hispanic white race, and higher prevalence of comorbidities). Despite our efforts to adjust for the potential confounders in the models, other residual confounders may still exist. During modeling, we found that after adjusting for age, the difference in eGFR levels between the smokers and non-smokers decreased. Older age played an important role in the development of adverse kidney function.

Among the non-smokers, sleep duration had a significant effect on eGFR. Either less or more sleep duration was harmful to the renal function, which was associated with a decline in eGFR. Normal sleep duration is an important profactor of the renal function in the non-smoking population. No 


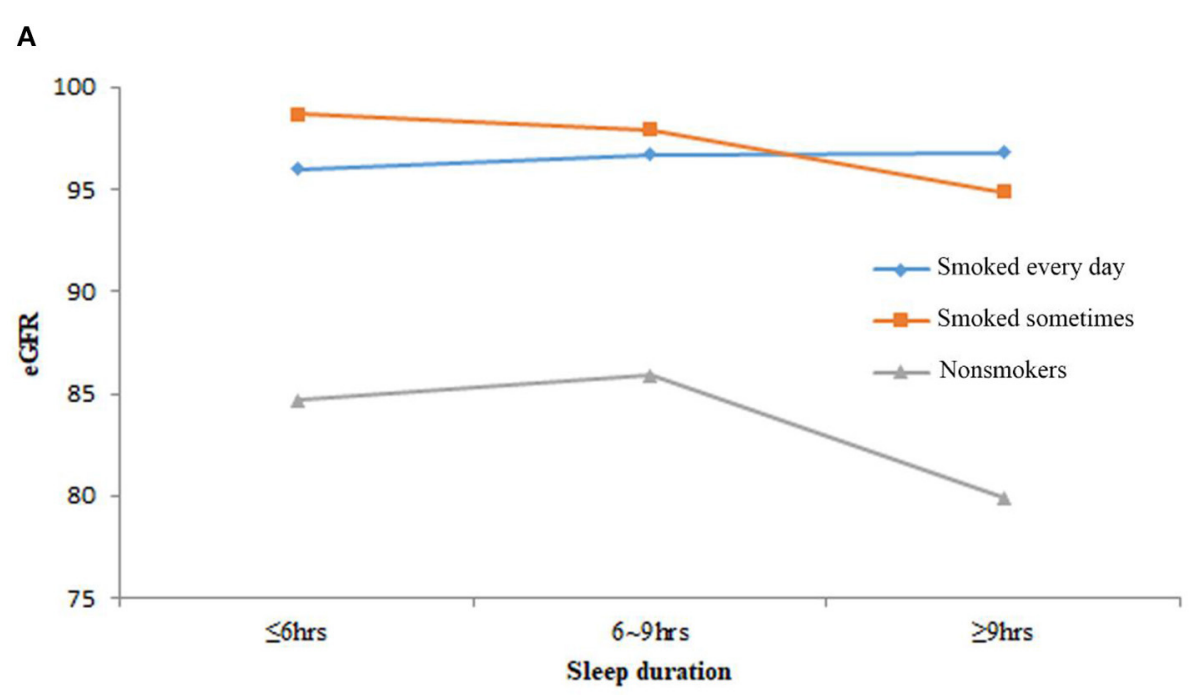

B

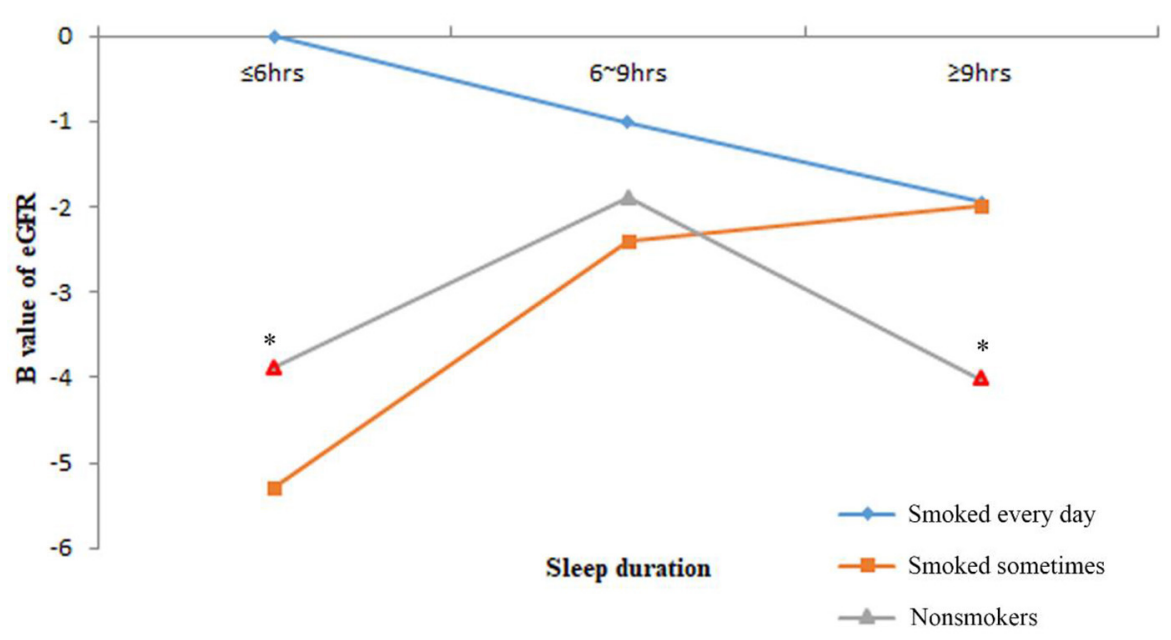

FIGURE 2 | The estimated glomerular filtration rate (eGFR) levels of the nine subgroups of study population by sleep duration and smoking frequency. (A) The average eGFR levels of nine subgroups. (B) The B value of eGFR in multivariable linear regression models after adjusting age, race, body mass index (BMI), marital status, frequency of snoring or breath cessation, diabetes, high blood pressure, high cholesterol, anemia, congestive heart failure, coronary heart disease and stroke. Marks in red border and asterisks denoted $p<0.05$ in the multivariable linear regression models.

statistical significance of interaction effect was found between sleep duration and smoking status on eGFR in the multivariable linear regression models. However, the effects of sleep duration on eGFR levels varied with the smoking frequency. The eGFR levels of edSmoking increased as the sleep duration decreased, whereas the eGFR levels of stSmoking increased as the sleep duration increased. The U-shape effects of eGFR levels were observed among the non-smokers; the group with normal sleep duration had the highest eGFR levels. To the best of our knowledge, this is the first study to examine the interaction of sleep duration and smoking status on eGFR. However, because the NHANES datasets are cross-sectional, we were unable to understand the causal effects. Moreover, a reciprocal and prospective relationship exists between smoking and sleeping problems (12), and further research is required to unravel whether renal function has a reciprocal effect on sleep as well.

\section{CONCLUSIONS}

The effects of sleep duration on the renal function varied with smoking frequency. The non-smokers with short or long sleep duration exhibited significantly lower eGFR levels compared with those who smoked every day and 


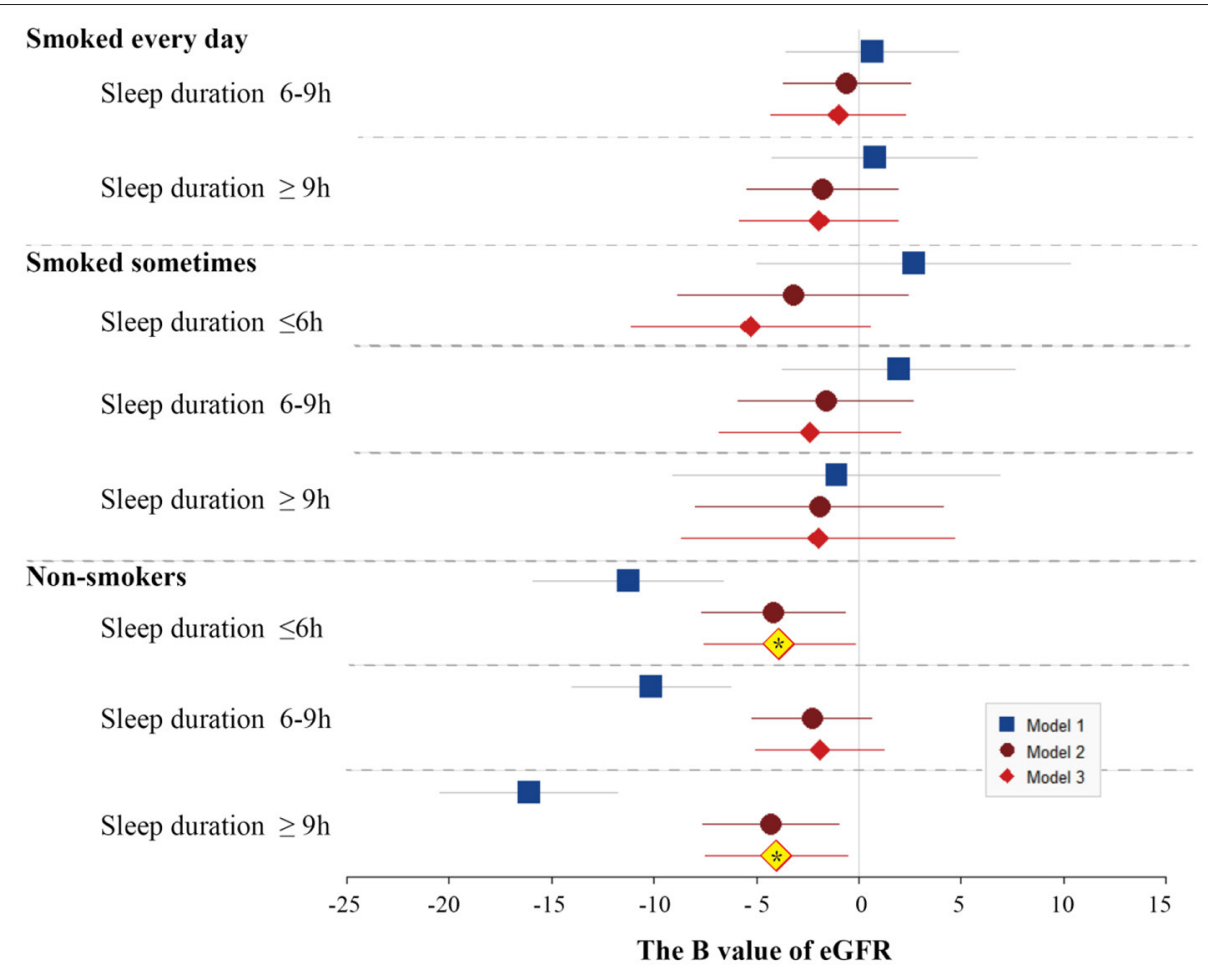

FIGURE 3 | Forest plots of B values of eGFR in the univariable and multivariable linear regression models among study population subgroups categorized by smoking and sleep duration. The subgroup of longer sleep duration and smoking every day was served as the reference group in the models. Model 1 was not adjusted. Model 2 was adjusted by age, race, BMl, and marital status. Model 3 was adjusted by age, race, BMl, marital status, frequency of snoring or breath cessation, diabetes, high blood pressure, high cholesterol, anemia, congestive heart failure, coronary heart disease, and stroke.

slept less after adjusting for demographic characteristics, sleep quality, and comorbidities. Normal sleep duration was a protective and more crucial factor for the nonsmokers than for smokers. As this was a cross-sectional study, further longitudinal studies are required to confirm the causal effects of sleep and smoking on the renal function.

\section{DATA AVAILABILITY STATEMENT}

Publicly available datasets were analyzed in this study. This data can be found at: National Health and Nutrition Examination Survey https://www.cdc.gov/nchs/nhanes/index.htm.

\section{REFERENCES}

1. Krishnamurthy S, Ks K, Dovgan E, Lustrek M, Gradisek Piletic B, Srinivasan $\mathrm{K}$, et al. Machine learning prediction models for chronic kidney disease using national health insurance claim data in Taiwan. Healthcare. (2021) 9:13. doi: 10.3390/healthcare9050546

2. Neborak JM, Mokhlesi B. Short sleep, sleep apnoea-associated hypoxaemic burden and kidney function: more questions than answers. Thorax. (2021) 78:638-9. doi: 10.1136/thoraxjnl-2020-216618

3. Belal N, Mutter C, Khanna D. Prevalence of sleep disorders in a population aged 16 and older. FASEB J. (2020) 34(S1). doi: 10.1096/fasebj.2020.34.s1.09932

\section{AUTHOR CONTRIBUTIONS}

Y-CL and Y-TC: data curation. Y-TC: formal analysis, software, and writing the original draft. $\mathrm{H}-\mathrm{EW}$ and $\mathrm{C}-\mathrm{CW}$ : resources. C-CW: supervision. H-EW, C-CW, Y-CL, C-MZ, PC, K-CL, and C-MC: writing, reviewing, and editing. All authors contributed to the article and approved the submitted version.

\section{FUNDING}

This study was supported by research grants from the Tri-Service General Hospital (TSGH-C05-110033) and the Ministry of Science and Technology (MOST110-2314-B-016-014), Taiwan.

4. Jaussent I, Cristol JP, Stengel B, Ancelin ML, Dupuy AM, Besset A, et al. Impact of sleep disturbances on kidney function decline in the elderly. Eur Respir J. (2016) 47:860-8. doi: 10.1183/13993003.01147-2015

5. Calero K, Anderson WM. Can poor sleep cause kidney disease? Another step closer to the answer. J Clin Sleep Med. (2019) 15:371-2. doi: 10.5664/jcsm.7652

6. Park S, Lee S, Kim Y, Lee Y, Kang MW, Kim K, et al. Short or long sleep duration and CKD: a mendelian randomization study. J Am Soc Nephrol. (2020) 31:2937-47. doi: 10.1681/ASN.2020050666

7. Knutson KL, Lash J, Ricardo AC, Herdegen J, Thornton JD, Rahman M, et al. Habitual sleep and kidney function in chronic kidney disease: the Chronic Renal Insufficiency Cohort study. J Sleep Res. (2018) 27:2819. doi: $10.1111 /$ jsr. 12573 
8. Lim SS, Vos T, Flaxman AD, Danaei G, Shibuya K, Adair-Rohani H, et al. A comparative risk assessment of burden of disease and injury attributable to 67 risk factors and risk factor clusters in 21 regions, 1990-2010: a systematic analysis for the Global Burden of Disease Study 2010. Lancet. (2012) 380:2224-60. doi: 10.1016/S0140-6736(12)61766-8

9. Choi HS, Han KD, Oh TR, Kim CS, Bae EH, Ma SK, et al. Smoking and risk of incident end-stage kidney disease in general population: a Nationwide Population-based Cohort Study from Korea. Sci Rep. (2019) 9:19511. doi: 10.1038/s41598-019-56113-7

10. Patten CA, Choi WS, Gillin JC, Pierce JP. Depressive symptoms and cigarette smoking predict development and persistence of sleep problems in US adolescents. Pediatrics. (2000) 106:E23. doi: 10.1542/peds.106.2.e23

11. Wong MM, Brower KJ, Zucker RA. Childhood sleep problems, early onset of substance use and behavioral problems in adolescence. Sleep Med. (2009) 10:787-96. doi: 10.1016/j.sleep.2008.06.015

12. Bellatorre A, Choi K, Lewin D, Haynie D, Simons-Morton B. Relationships between smoking and sleep problems in black and white adolescents. Sleep. (2017) 40:8. doi: 10.1093/sleep/zsw031

13. Centers for Disease Control Prevention (CDC), National Center for Health Statistics (NCHS), MD: U.S. Department of Health and Human Services CfDCa. National Health and Nutrition Examination Survey Data. (2021). Available online at: https://www.cdc.gov/nchs/nhanes/index.htm

14. Inker LA, Schmid CH, Tighiouart H, Eckfeldt JH, Feldman HI, Greene T, et al. Estimating glomerular filtration rate from serum creatinine and cystatin C. $N$ Engl J Med. (2012) 367:20-9. doi: 10.1056/NEJMoa1114248

15. Stevens LA, Levey AS. Measured GFR as a confirmatory test for estimated GFR. J Am Soc Nephrol. (2009) 20:2305-13. doi: 10.1681/ASN.2009020171

16. $\mathrm{R}$ Core Team. $R: A$ Language and Environment for Statistical Computing (2020).

17. Peterson RA, Cavanaugh JE. Ordered quantile normalization: a semiparametric transformation built for the cross-validation era. J Appl Stat. (2019) 47:1-16. doi: 10.1080/02664763.2019.1630372

18. Farhat A, Jones IA, Saadat S, Dornhofer K, Kong C, Nguyen T, et al. The association of smoking with ultrasound-measured kidney dimensions. Clin Nephrol. (2020) 93:9-16. doi: 10.5414/CN109854

19. Jain G, Jaimes EA. Nicotine signaling and progression of chronic kidney disease in smokers. Biochem Pharmacol. (2013) 86:1215-23. doi: 10.1016/j.bcp.2013.07.014

20. Leonberg-Yoo AK, Rudnick MR. Tobacco use: a chronic kidney disease accelerant. Am J Nephrol. (2017) 46:257-9. doi: 10.1159/000481209

21. Orth SR. Cigarette smoking: an important renal risk factor - far beyond carcinogenesis. Tob Induc Dis. (2002) 1:13755. doi: 10.1186/1617-9625-1-2-137

22. Arany I, Grifoni S, Clark JS, Csongradi E, Maric C, Juncos LA. Chronic nicotine exposure exacerbates acute renal ischemic injury. Am J Physiol Renal Physiol. (2011) 301:F125-33. doi: 10.1152/ajprenal.00041.2011

23. Sanner T, Grimsrud TK. Nicotine: carcinogenicity and effects on response to cancer treatment - a review. Front Oncol. (2015) 5:196. doi: 10.3389/fonc.2015.00196

24. Lee S, Kang S, Joo YS, Lee C, Nam KH, Yun HR, et al. Smoking, smoking cessation, and progression of chronic kidney disease: results from KNOWCKD study. Nicotine Tob Res. (2021) 23:92-8. doi: 10.1093/ntr/ntaa071

25. Ramalingam A, Santhanathas T, Shaukat Ali S, Zainalabidin S. Resveratrol supplementation protects against nicotine-induced kidney injury. Int $J$ Environ Res Public Health. (2019) 16:13. doi: 10.3390/ijerph16224445

26. Yamazaki H, Horiuchi K, Takano R, Nagano T, Shimizu M, Kitajima M, et al. Human blood concentrations of cotinine, a biomonitoring marker for tobacco smoke, extrapolated from nicotine metabolism in rats and humans and physiologically based pharmacokinetic modeling. Int J Environ Res Public Health. (2010) 7:3406-21. doi: 10.3390/ijerph7093406

27. Nakajima M, Yokoi T. Interindividual variability in nicotine metabolism: Coxidation and glucuronidation. Drug Metab Pharmacokinet. (2005) 20:22735. doi: $10.2133 / \mathrm{dmpk} .20 .227$

28. Kunutsor SK, Spee JM, Kieneker LM, Gansevoort RT, Dullaart RPF, Voerman AJ, et al. Self-reported smoking, urine cotinine, and risk of cardiovascular disease: findings from the PREVEND (Prevention of Renal and Vascular End-Stage Disease) Prospective Cohort Study. J Am Heart Assoc. (2018) 7:15. doi: 10.1161/JAHA.118.008726
29. Hellemons ME, Sanders JS, Seelen MA, Gans RO, Muller Kobold AC, van Son WJ, et al. Assessment of cotinine reveals a dose-dependent effect of smoking exposure on long-term outcomes after renal transplantation. Transplantation. (2015) 99:1926-32. doi: 10.1097/TP.0000000000000636

30. Torres S, Merino C, Paton B, Correig X, Ramirez N. Biomarkers of exposure to secondhand and thirdhand tobacco smoke: recent advances and future perspectives. Int J Environ Res Public Health. (2018) 15:25. doi: 10.3390/ijerph15122693

31. Rezonzew G, Chumley P, Feng W, Hua P, Siegal GP, Jaimes EA. Nicotine exposure and the progression of chronic kidney disease: role of the alpha7nicotinic acetylcholine receptor. Am J Physiol Renal Physiol. (2012) 303:F30412. doi: 10.1152 /ajprenal.00661.2011

32. Halimi JM, Giraudeau B, Vol S, Caces E, Nivet H, Lebranchu Y, et al. Effects of current smoking and smoking discontinuation on renal function and proteinuria in the general population. Kidney Int. (2000) 58:128592. doi: $10.1046 /$ j.1523-1755.2000.00284.x

33. Arany I, Hall S, Dixit M. Age-dependent sensitivity of the mouse kidney to chronic nicotine exposure. Pediatr Res. (2017) 82:822-8. doi: 10.1038/pr.2017.153

34. Ohkuma T, Nakamura U, Iwase M, Ide H, Fujii H, Jodai T, et al. Effects of smoking and its cessation on creatinine- and cystatin C-based estimated glomerular filtration rates and albuminuria in male patients with type 2 diabetes mellitus: the Fukuoka Diabetes Registry. Hypertens Res. (2016) 39:744-51. doi: 10.1038/hr.2016.51

35. Akomolafe OR, Imafidon CE, Olukiran OS, Oladele AA, Akanji BO. Subacute administration of lower doses of nicotine caused sex-dependent improvement of renal function in Wistar rats. Toxicol Rep. (2017) 4:53542. doi: 10.1016/j.toxrep.2017.10.001

36. Riveles K, Huang LZ, Quik M. Cigarette smoke, nicotine and cotinine protect against 6-hydroxydopamine-induced toxicity in SH-SY5Y cells. Neurotoxicology. (2008) 29:421-7. doi: 10.1016/j.neuro.2008.02.001

37. Moran VE. Cotinine: beyond that expected, more than a biomarker of tobacco consumption. Front Pharmacol. (2012) 3:173. doi: 10.3389/fphar.2012.00173

38. Sadis C, Teske G, Stokman G, Kubjak C, Claessen N, Moore F, et al. Nicotine protects kidney from renal ischemia/reperfusion injury through the cholinergic anti-inflammatory pathway. PloS one. (2007) 2:e469. doi: 10.1371/journal.pone.0000469

39. Lee HJ, Kwak N, Kim YC, Choi SM, Lee J, Park YS, et al. Impact of sleep duration on mortality and quality of life in chronic kidney disease: results from the 2007-2015 KNHANES. Am J Nephrol. (2021) 52:18. doi: $10.1159 / 000516096$

40. Hao Q, Xie M, Zhu L, Dou Y, Dai M, Wu Y, et al. Association of sleep duration with chronic kidney disease and proteinuria in adults: a systematic review and dose-response meta-analysis. Int Urol Nephrol. (2020) 52:130520. doi: 10.1007/s11255-020-02488-w

41. Tan NYQ, Chan J, Cheng CY, Wong TY, Sabanayagam C. Sleep duration and diabetic kidney disease. Front Endocrinol. (2018) 9:808. doi: 10.3389/fendo.2018.00808

42. Bo Y, Yeoh EK, Guo C, Zhang Z, Tam T, Chan TC, et al. Sleep and the risk of chronic kidney disease: a cohort study. J Clin Sleep Med. (2019) 15:393-400. doi: $10.5664 /$ jcsm. 7660

43. Hui L, Benca R. The bidirectional relationship between obstructive sleep apnea and chronic kidney disease. J Stroke Cerebrovasc Dis. (2021) 30:105652. doi: 10.1016/j.jstrokecerebrovasdis.2021.105652

44. Wang Y, Meagher RB, Ambati S, Cheng H, Ma P, Phillips BG. Patients with obstructive sleep apnea have altered levels of four cytokines associated with cardiovascular and kidney disease, but near normal levels with airways therapy. Nat Sci Sleep. (2021) 13:457-66. doi: 10.2147/NSS.S282869

45. Meng L, Ding Y, Li J, Li X, Yan T, Yang M, et al. Impact of inflammatory markers on the relationship between sleep quality and diabetic kidney disease. Sleep Breath. (2021). doi: 10.1007/s11325-021-02380-6. [Epub ahead of print].

46. Jackson CL, Umesi C, Gaston SA, Azarbarzin A, Lunyera J, McGrath JA, et al. Multiple, objectively measured sleep dimensions including hypoxic burden and chronic kidney disease: findings from the Multi-Ethnic Study of Atherosclerosis. Thorax. (2020) 76:704-13. doi: 10.1136/thoraxjnl-2020-214713

47. Jhamb M, Ran X, Abdalla H, Roumelioti ME, Hou S, Davis H, et al. Association of sleep apnea with mortality in patients with advanced kidney 
disease. Clin J Am Soc Nephrol. (2020) 15:182-90. doi: 10.2215/CJN.078 80719

48. Gibson M, Munafo MR, Taylor AE, Treur JL. Evidence for genetic correlations and bidirectional, causal effects between smoking and sleep behaviors. Nicotine Tob Res. (2019) 21:731-8. doi: 10.1093/ntr/nty230

49. Li H, Liu Y, Xing L, Yang X, Xu J, Ren Q, et al. Association of cigarette smoking with sleep disturbance and neurotransmitters in cerebrospinal fluid. Nat Sci Sleep. (2020) 12:801-8. doi: 10.2147/NSS.S272883

50. Dugas EN, Sylvestre MP, O'Loughlin EK, Brunet J, Kakinami L, Constantin E, et al. Nicotine dependence and sleep quality in young adults. Addict Behav. (2017) 65:154-60. doi: 10.1016/j.addbeh.2016.10.020

51. Mathews HL, Stitzel JA. The effects of oral nicotine administration and abstinence on sleep in male C57BL/6J mice. Psychopharmacology. (2019) 236:1335-47. doi: 10.1007/s00213-018-5139-6

52. Speeckaert MM, Delanghe JR, Vanholder RC. Chronic nicotine exposure and acute kidney injury: new concepts and experimental evidence. Nephrol Dial Transplant. (2013) 28:1329-31. doi: 10.1093/ndt/gft019

53. Cooper RG. Effect of tobacco smoking on renal function. Indian J Med Res. (2006) 124:261-8.
Conflict of Interest: The authors declare that the research was conducted in the absence of any commercial or financial relationships that could be construed as a potential conflict of interest.

Publisher's Note: All claims expressed in this article are solely those of the authors and do not necessarily represent those of their affiliated organizations, or those of the publisher, the editors and the reviewers. Any product that may be evaluated in this article, or claim that may be made by its manufacturer, is not guaranteed or endorsed by the publisher.

Copyright (c) 2021 Wu, Wang, Liu, Zheng, Chu, Lu, Chu and Chang. This is an open-access article distributed under the terms of the Creative Commons Attribution License (CC BY). The use, distribution or reproduction in other forums is permitted, provided the original author(s) and the copyright owner(s) are credited and that the original publication in this journal is cited, in accordance with accepted academic practice. No use, distribution or reproduction is permitted which does not comply with these terms. 